\title{
Sécurité et confiance
}

\section{Barbara Züst}

lic. iur. HSG, Co-directrice OSP Organisation Suisse des Patients

Les maladies sont une source d'inquiétude physique et psychologique. Le besoin de réconfort et de sécurité est donc l'une des principales préoccupations des patients. Un patient sécurisé accordera plus facilement sa confiance au personnel médical. Mais l'inverse est également vrai: le comportement adéquat des professionnels suscitera la confiance du patient. La confiance, c'est d'avoir le sentiment que les prestations qui me sont prodiguées le sont véritablement dans mon intérêt. Mais c'est aussi de savoir que dans le cadre de l'anamnèse, mes observations de la maladie et les informations que je communique au médecin jouent un rôle majeur. Sur le plan diagnostique notamment, le patient contribue largement au succès thérapeutique. C'est ce qu'a très clairement démontré un nouveau cas récent particulièrement tragique qui nous avait été soumis pour examen [1].

Un jeune trentenaire qui venait de rentrer en Suisse après un séjour de trois semaines dans son pays d'origine en Europe de l'Est consulte un médecin en raison de douleurs persistantes à la nuque et à la tête ainsi que d'une sensibilité à la lumière. Il lui expose son épisode

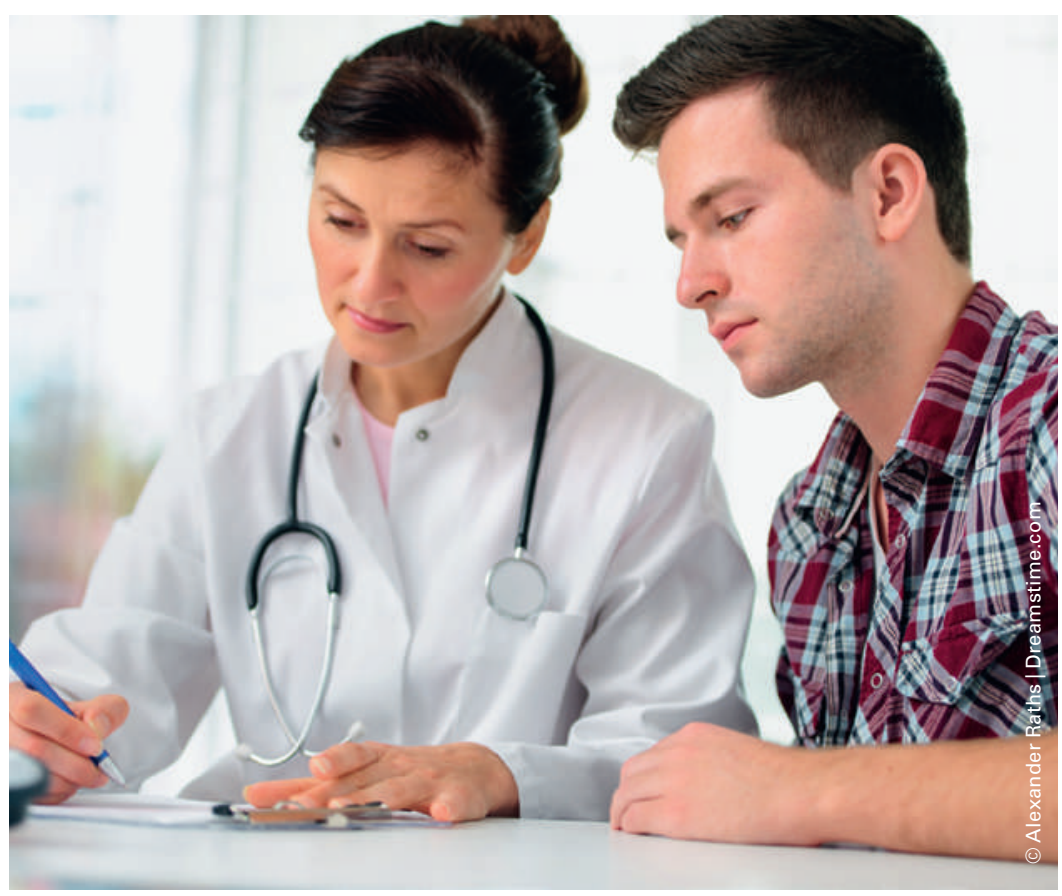

Une relation de confiance est importante pour que la communication entre médecin et patient puisse fonctionner. fiévreux, ses trois vomissements ainsi que la vision double dont il souffre depuis plusieurs jours. Le scanner du crâne ne révèle aucune anomalie. Après une nuit passée en observation et l'administration de médicaments antalgiques, les médecins constatent une amélioration et le laissent rentrer chez lui. Une dizaine de jours plus tard, il signale de nouveaux maux de tête, des nausées, des vomissements, une sensibilité à la lumière et une tachycardie inquiétante. Après divers examens (examen cardiaque et IRM du crâne), les médecins traitants supposant qu'il s'agit d'une maladie inflammatoire et non infectieuse lui prescrivent de la cortisone. Ce n'est que trois jours plus tard que des antibiotiques lui sont administrés en complément, notamment en raison de taux croissants de leucocytes, de lactate et de liquide céphalorachidien.

Moins de dix jours après son hospitalisation, le patient perd soudainement conscience et subit un arrêt respiratoire. Les mesures de réanimation restent vaines. L'autopsie révèle alors une méningite avec des abcès au cervelet et au tronc cérébral. Malgré d'intensives recherches - y compris la détection de matériel génétique de bactéries spécifiques -, l'agent pathogène demeure inconnu.

Ce n'est qu'après ce décès tragique que des échanges avec les proches ont révélé le traitement dentaire que le jeune homme avait subi dans son pays d'origine suite à des maux de dents. C'était à ce moment-là que les maux de tête et les troubles décrits plus haut étaient apparus. Suspectant une méningite, le médecin consulté sur place avait voulu lui administrer des antibiotiques. Mais le jeune homme avait préféré se faire soigner en Suisse. A posteriori, il est clair que le manque de communication - malgré la transmission du dossier médical rédigé en langue étrangère - a largement contribué au déroulement tragique.

Les médecins savent combien l'anamnèse est importante pour établir le diagnostic: une réalité souvent méconnue du patient qui apprécie d'être instruit dans ce sens. Tous les progrès techniques de la médecine ne remplaceront jamais l'échange d'informations entre le patient et son médecin. L'anamnèse et les informations recueillies auprès de tiers demandent certes du temps, mais permettent souvent un important gain de temps ultérieur. 
Nous encourageons donc les patients à jouer un rôle proactif et leur donnons, à titre d'exemple, les recommandations suivantes [2]:

- lorsque le médecin interroge le patient sur ses antécédents médicaux, le moindre détail peut être important. Le patient doit donc communiquer absolument le moindre fait, même s'il ne lui revient à l'esprit qu'après le premier entretien;

- si le patient a l'impression de ne pas être pris au sérieux, il doit impérativement en parler directement à l'équipe thérapeutique;

- si ses symptômes évoluent en cours de consultation, il doit impérativement en informer le médecin - même si ces changements surviennent au moment de sa sortie ou de son transfert vers un autre service;

- au moment de la planification des prochaines étapes comme des examens complémentaires, une hospitalisation ou la sortie, le patient devrait demander un complément d'information: Pourquoi est-il nécessaire d'effectuer un examen complémentaire? Quels sont les risques? Pourquoi une hospitalisation est-elle nécessaire? Une sortie avec un risque acceptable est-elle envisageable?

- il peut être utile pour le patient de soumettre ses souhaits et ses questions au médecin par écrit;

- il est préférable dans une telle situation que le patient soit soutenu par un proche. Attention: la désignation provisoire d'une maladie lors d'une consultation d'urgence peut s'avérer erronée ultérieurement. Il est donc primordial que le patient se renseigne dans quels cas ou dans quelle évolution de son état il doit se manifester.

Ces différents points relèvent bien évidemment du devoir d'information du médecin. Néanmoins, des patients attentifs peuvent largement contribuer à leur sécurité, dès lors que leur état de santé le permet.

\section{Obstacles à la communication}

Le déroulement tragique du traitement de ce jeune homme montre de manière saisissante combien l'échange sans réserve d'informations entre le médecin et son patient est essentiel dans le cadre de l'anamnèse. Des connaissances linguistiques insuffisantes constituent visiblement un obstacle insurmontable. Les autres raisons pour lesquelles le patient garde pour lui des informations importantes ou n'en communique que des fragments sont en revanche plus difficiles à déceler. Par analogie au phénomène de l'institution totale [3] selon Erving Goffmann [4], l'institution «hôpital» à elle seule ainsi que son environne- ment - par ex. la répartition claire des rôles, le port de l'uniforme médical, etc. - peuvent affecter l'identité du patient. Cela explique pourquoi des personnes dotées d'une certaine confiance en elles deviennent parfois timides à l'hôpital et n'osent pas se renseigner auprès du personnel médical en cas de doute.

\section{Contribution du patient à la sécurité}

Nous avons besoin d'une culture qui encourage un comportement actif et participatif du patient. La Suisse n'est pas le seul pays à constater le désir accru des patients de participer plus activement; au plan international, le "patient-apprenant» est également en plein développement. C'est ce qu'illustre par exemple le dépliant Involve Yourself conçu à l'University of California ou le manuel danois dédié aux patients $A$ patient's guide to a safer hospital stay [5]

Une culture du patient proactif implique de témoigner de l'estime aux patients lorsque ces derniers se révèlent des interlocuteurs attentifs, et de demander des précisions sur les points obscurs. Voici un exemple frappant qui nous a été rapporté par une patiente: venue se faire administrer la troisième injection contre la rage dans le cabinet de son médecin de famille, elle a immédiatement réalisé que l'assistante médicale n'avait pas saisi l'ampoule «Rabipur» habituelle, mais une autre préparation destinée à la patiente suivante. L'attention de la patiente a donc permis d'éviter une erreur.

L'important, c'est de montrer aux patients que leur contribution est la bienvenue puisqu'elle permet d'accroître la sécurité, et que les questions posées ne sont pas ressenties comme un acte de méfiance.

A l'Organisation suisse des patients (OSP), nous sensibilisons nous aussi les patients, à travers notre guide complet Patientenkompass, aux mesures susceptibles de contribuer à leur sécurité [6]. Comme nous l'avons mentionné au début, une personne qui se sent en sécurité accordera, en effet, plus facilement sa confiance. Et la confiance est le fondement d'une bonne relation entre le patient et son médecin.

\section{Références}

1 Nous nous contentons d'esquisser les grandes lignes pour des raisons de place.

2 Guides/Bulletin d'information OSP-Actualités, Daniel Tapernoux, med. pract., 2013/4, disponible sur www.spo.ch (en allemand).

3 https://fr.wikipedia.org/wiki/Institution totale

4 Sociologue canadien, 1922-1982.

5 Bachinger G. Die Beteiligung des Patienten: der lernende Patient. Aus Patientenperspektive, $1^{\text {re }}$ éd. 2012; p. 39.

6 Züst B. Der Patientenkompass, $1^{\text {re }}$ éd.; Zurich: Xanthippe Verlag; 2015. En allemand (traduction en cours). A commander sur www. spo.ch 